\title{
The influence of viewing conditions on radiological diagnosis of periapical inflammation
}

\author{
N. Patel, ${ }^{1}$ V. E. Rushton, ${ }^{2}$ T. V. Macfarlane, ${ }^{3}$ and K. Horner, ${ }^{4}$
}

\begin{abstract}
Objectives To determine the effect of viewing conditions upon diagnosis of early periapical inflammatory pathosis on intra-oral radiographs, and to examine the effect of observer experience upon diagnostic performance in this task.

Methods 50 observers examined 18 periapical radiographs using three different viewing conditions (room lighting; viewing box; viewing box with $\mathrm{x} 2$ magnification and masking). Their diagnoses were compared with an 'expert' diagnosis provided by repeated viewings of the films by two dental radiologists. Sensitivities and specificities were determined.

Results When 'ideal' viewing conditions were used, optimal sensitivity $(78 \%)$ and specificity $(78 \%)$ were obtained. Use of a viewing box was associated with significantly higher specificity than the use of room lighting $(P=0.0016)$. Use of masking and $\mathrm{x} 2$ magnification was associated with significantly higher sensitivity than a viewing box alone $(P=0.004)$. There were few significant differences in diagnostic performance between observers, but qualified dental staff had significantly higher specificities than 4 th year $(P=0.01)$ and 5 th year $(P=0.01)$ students when a viewing box was used alone.

Conclusions This study on early periapical inflammatory pathosis gives support to guidelines which recommend the use of a viewing box, $\mathrm{x} 2$ magnification and masking for interpreting intra-oral radiographs. It also suggests that observer experience may influence interpretation of early periapical pathosis.
\end{abstract}

$\mathrm{R}$ adiography plays an important part in the diagnosis of periapical inflammatory pathosis. While most dentists are aware of the importance of standardising exposure and processing conditions to enable the production of an image of optimal diagnostic quality, ${ }^{1}$ most fail to use 'ideal' viewing conditions for interpretation of the radiograph. ${ }^{2-6}$ This is mainly caused by the lack of an adequate viewing box or magnifier.

A number of reports have emphasised the value of using a viewing box,${ }^{7-11}$ magnification facility ${ }^{7,8,11}$ and masking of extraneous light $^{7-9,11,12}$ when interpreting intra-oral radiographs. In the UK, the routine use of 'ideal viewing conditions' have been recommended in a set of influential guidelines. ${ }^{1}$ Moreover, in a recent review paper ${ }^{13}$ outlining error and variation in interpretation of the radiographic image, the standardisation of viewing conditions has been proposed as one of several strategies to optimise

\footnotetext{
${ }^{1}$ Final Year Dental Student, ${ }^{2}$ Lecturer in Dental and Maxillofacial Radiology, ${ }^{3}$ Lecturer in Dental Statistics, ${ }^{4}$ Senior Lecturer in Dental and Maxillofacial Radiology, Turner Dental School, University Dental Hospital of Manchester, Higher Cambridge Street, Manchester M15 6FH

Correspondence to: VE Rushton

REFEREED PAPER

Received 09.11.99; accepted 09.02.00

(C) British Dental Journal 2000; 189: 40-42
}

perception and consequently interpretation.

Apart from one small study, ${ }^{10}$ limited information is available about the degree to which these viewing conditions influence performance in the diagnosis of periapical inflammatory pathosis on intra-oral radiographs.

The aims of this study were:

1. To determine the effect of viewing conditions upon diagnostic accuracy in interpretation of early periapical inflammatory pathosis on intra-oral radiographs, and

2. To examine the influence of observer experience upon diagnostic accuracy in this task.

\section{Materials and methods}

Two of the authors, a Consultant and a Lecturer in Dental and Maxillofacial Radiology, searched the film library of a dental Hospital radiology department and identified, using consensus methods, ${ }^{14} 50$ teeth with normal periapical anatomy and 50 which each demonstrated a tooth with early periapical inflammatory changes. The latter were defined as a widening of the periodontal ligament space (PDL), a loss of periapical lamina dura or a combination of both. These signs have been found to be the most important radiographic features influencing differentiation between healthy teeth and those with periapical inflammation. ${ }^{15}$ The method of consensus decision making was simultaneous viewing of radiographs by the two observers under the following conditions: a standard $30 \mathrm{~cm}$ by $15 \mathrm{~cm}$ dental viewing box, $\mathrm{x} 2$ magnification and masking of peripheral light using opaque card. High intensity illumination was not used, but any radiograph that was considered to have excessive density was excluded without further consideration. The room lighting was dimmed. Radiographs were excluded from possible use in the study where there was anything other than complete agreement on the presence of periapical inflammation. From the pool of 100 radiographs, 20 were selected in this manner, consisting of:

- Five anterior (incisor or canine) teeth with normal periapical anatomy

- Five anterior teeth with early periapical pathosis

- Five posterior (premolar or molar) teeth with normal periapical anatomy

- Five posterior teeth with early periapical pathosis.

All radiographs, although from differing film batches, were from the same manufacturer (Kodak) and of the same speed (Ektaspeed plus) and had been processed using standardised processing conditions. This selection process excluded radiographs with unacceptable deficiencies in quality.

In order that 'clues' about the health of the teeth (such as large restorations or caries) would not influence decisions of observers, the crowns of the teeth on each radiograph were obscured with 
black tape. The 20 radiographs were randomly numbered.

After a 1-week interval the radiographs were viewed again by the same two observers and each tooth classified as 'normal' or showing signs of early periapical inflammatory pathosis. There was agreement between second and initial diagnosis for all but two radiographs. These two were then excluded from the study The remaining 18 radiographs for which diagnosis had been confirmed at the second viewing were retained. The 'expertderived' diagnoses for these teeth were subsequently used as the basis for the study.

The 18 radiographs were then examined by 50 observers (each in isolation) with a wide variety of clinical backgrounds. The observers consisted of 11 fourth year dental students, 25 fifth year dental students and 14 dental staff of the dental school. Each observer viewed the radiographs independently on three occasions, separated by at least a 2 -week interval, with different viewing conditions:

\section{Using normal room lighting only}

2. Using a standard viewing box, with no magnification or masking

3. Using a standard viewing box with $\mathrm{x} 2$ magnification and complete masking of peripheral light.

The viewing boxes used in the study had cleaned viewing surfaces, fluorescent tube replacement, and lightmeter and photometer analysis to exclude any boxes with gross mismatches in either colour, brightness or variation in intensity. ${ }^{16,17}$

For each radiograph, on each occasion, the observers were asked to record whether the tooth showed normal periapical anatomy or evidence of early periapical inflammatory changes. To further avoid the chance that an observer might remember a previous diagnosis, the numbering of radiographs was reallocated after each viewing.

The diagnostic performance of each observer for each of the three viewing conditions was determined by calculation of sensitivity and specificity using the 'expert-derived' diagnosis by the two dental radiologists as the truth. Analyses were performed using SPSS $^{18}$ and STAT ${ }^{19}$ statistical software. Data from different observers were grouped as required for statistical analysis according to viewing conditions and observer (4th year student, 5 th year student, and staff) to determine any differences in diagnostic performance. Wilcoxon signed ranks test and Mann-Whitney tests were used to compare sensitivities and specificities of different viewing condition/observer combinations. The significance level was set at the $\alpha=0.02$ level to adjust for multiple comparisons. ${ }^{20}$ Kappa statistic was used to measure the agreement between observers for each viewing condition.

\section{Results}

The median sensitivities and specificities for all 50 observers are shown in Table 1. The use of a viewing box with magnification and masking of peripheral light gave the best combination of sensitivity $(77.8 \%)$ and specificity (77.8\%). Furthermore, these conditions were associated with significantly better sensitivity than with the viewing box $(P=0.004)$, and significantly better specificity than with room lighting alone $(P=0.016)$.

When one compares between different levels of expertise, the only significant differences were seen between staff and students for specificity when observing radiographs using a viewing box. The staff performed significantly better than either 4 th year students $(P=0.01)$ or 5 th year students $(P=0.01)$. There was only fair agreement between all the observers $(\mathrm{kappa}=0.26)$ in radiological interpretation of periapical status.

\section{Discussion}

This study used radiographs of teeth with early evidence of periapical pathosis. It was assumed that while larger periapical lesions are relatively simple to identify, correct diagnosis of more subtle

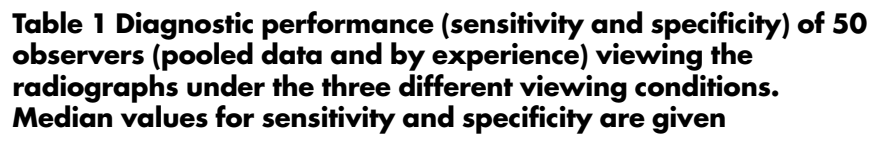

Viewing conditions Median sensitivity (\%) Median specificity(\%)

\section{Room lighting}

$\begin{array}{lll}\text { 4th year } & 77.8 & 66.7 \\ \text { 5th year } & 66.7 & 66.7 \\ \text { Staff } & 72.2 & 77.8 \\ \text { Overall } & 66.7 & 72.2\end{array}$

\section{Viewing box}

4 th year

5th year

Staff

66.7

66.7

Overall

$\begin{array}{ll}66.7 & 77.8\end{array}$

66.7

88.9

Viewing box, magnification

and masking

4th year

5 th year

Staff

Overall

$\begin{array}{ll}66.7 & 66.7 \\ 88.9 & 77.8 \\ 72.2 & 77.8 \\ 77.8 & 77.8\end{array}$

early inflammatory changes would be more likely to be influenced by viewing conditions and be more appropriate for the study. The 'expert-derived' diagnosis could be criticised in the absence of histological confirmation of the periapical status. ${ }^{21,22}$ However, the method of arriving at this diagnosis, comprising initial consensus selection by 'expert' observers ${ }^{14}$ followed by repeat examination to exclude equivocal diagnoses, was considered a 'best there is' technique. An alternative, and more rigorous, method could have employed radiographs of teeth from cadaver jaws followed by sectioning and histological analysis of each tooth's periapex to determine a gold standard diagnosis. However, this was considered impracticable bearing in mind the difficulties in obtaining cadaver material.

In the diagnosis of periapical pathosis a dentist uses clinical findings and the radiographic findings to reach a diagnosis. Thus this study, where radiographs were used in isolation, could be accused of being somewhat divorced from real clinical practice. It would be useful to repeat the study, but with the addition of particular clinical information such as the tooth vitality, to determine whether the diagnostic performance of observers change. However, we considered it important initially to look at radiological diagnosis alone. To reinforce this, the crowns of the teeth on the radiographs were obscured so that possible indicators of periapical pathosis such as caries or large restorations were not available to the observers.

Sensitivity (true positive diagnoses) and specificity (true negative diagnoses) are both important in radiographic diagnosis. Indeed, in the context of early periapical inflammatory changes in dental practice, it could be argued that specificity is the more important. While deficiencies in sensitivity will simply lead to an asymptomatic lesion being missed, perhaps to be detected when symptomatic or larger radiographically, poor specificity might lead to unnecessary endodontic treatment.

The results showed that significantly higher mean specificity was seen with a shift from ordinary room lighting to using a viewing box. The 'ideal' viewing conditions (viewing box, magnification and masking) gave the best combination of sensitivity and specificity. Thus this study supports guidelines which recommend these for general dental practice.

The fundamental differences between the three sets of viewing conditions were degree and source of illumination, image size and masking of extraneous light, all of which affect the perceptibility of image contrast. $9,23,24$

For example, reduced light intensity, such as might occur when 
viewing films with ambient room lighting, gives a subjective reduction in image contrast and an increase in average density. ${ }^{24}$ When viewing a small film, say a periapical, on a larger viewing box the eyes are affected by the light reaching it from around the film. This subjectively increases film blackening and reduces perceived contrast. This 'glaring' effect can also occur when viewing radiographs held up to sunlight through a window or against a bright dental operating light. By eliminating extraneous light and complete masking, the perceived contrast is increased. ${ }^{9}$

These observed effects of different viewing conditions may be explained by consideration of the response of the eye to light. The rods and cones of the retina are found over its entire surface apart from the fovea, where only cones are present. Cones are primarily used for colour vision while rods only interpret information as black, white or shades of grey. On exposure to bright light the visual pigment migrates between the cones, stopping the spread of light from one cone to another. This improves visual acuity (the ability to differentiate between two objects with a minimum distance between them). When exposed to dim light, visual acuity is decreased because the visual pigment remains within the rods and cones allowing light to spread between them. While this increases the sensitivity, it reduces acuity. As the light becomes dimmer, the cones stop functioning and the rods take over, leading to further reduction in acuity.

We chose the three groups of observers for the study because we anticipated that clinical experience might be an influential factor in diagnostic performance. At our dental school, students receive instruction in radiological interpretation during the fourth year. At the time of the study, all fourth year students had completed that section of the course dealing with interpretation of normal periapical anatomy and periapical inflammation. Comparison of the three observer groups gave few significant findings, namely that the specificity of staff diagnoses was significantly higher than that of both student groups when a viewing box was used. It can be concluded that education influences specificity, a finding that has been demonstrated in previous research on occlusal caries diagnosis. ${ }^{25}$ This may reflect a tendency of experienced clinicians only to make a positive diagnosis where they have greater confidence. Practically, as discussed above, the consequences of missing an asymptomatic small periapical lesion are minimal, and greater clinical experience may lead to a more cautious approach. ${ }^{26,27}$

While statistically significant differences between the three groups of observers were few, a noticeable finding was a wide variation in diagnostic performance between observers, with only fair agreement seen. Such a wide variation in observer performance in periapical radiological diagnosis is widely recognised. ${ }^{28-31}$

In the study, we did not address the issue of variation in experience and performance within the staff group. In retrospect it would have been interesting to assess whether specialist staff (such as consultants in restorative dentistry and endodontic specialists) might have greater expertise than more junior dentists and those in other specialties.

\section{Conclusion}

This study on early periapical inflammatory pathosis gives support to guidelines which recommend the use of a viewing box, magnification and masking for interpreting intra-oral radiographs. It also suggests that observer experience influences performance in the interpretation of periapical pathosis.

The authors would like to thank all the students and staff who participated in this study.
1 Royal College of Radiologists and National Radiological Protection Board. Guidelines on radiology standards for primary dental care. Chilton: National Radiological Protection Board, 1994, Documents of the NRPB; 5: p24.

2 Pentel L, Goren A D, Shapiro G et al. A survey of dental x-ray equipment and radiological practices in Nassau County, New York. Health Phys 1971; 20: 59-71.

3 Rout P J, Cook C. Survey of dental radiography in general dental practice.J Dent 1982; 10: 12-16.

4 Kaugars G E, Broga G W, Collett W K. Dental radiologic survey of Virginia and Florida. Oral Surg Oral Med Oral Pathol 1985; 60: 225-229.

5 Monsour P A, Kruger B J. X-ray equipment used by general dental practitioners in Australia. Aust Dent J 1988; 33: 81-86.

6 Szymkoviak L E, Sarll D W, Horner K. Some factors affecting the standards of radiography in general dental practice. Br Dent J 1995; 179: 168-174.

7 Wuehrmann A H. Radiation hygiene and its practice in dentistry as related to film viewing procedures and radiographic interpretation. J Am Dent Assoc 1970; 80: 346-356.

8 Brynolf I. Improved viewing facilities for better roentgenodiagnosis. Oral Surg 1971; 32: 808-811.

9 Welander U, McDavid W D, Higgins, N M et al. The effect of viewing conditions on the perceptibility of radiographic details. Oral Surg Oral Med Oral Pathol 1983; 56: 651-654.

10 Antrim D D. Reading the radiograph: a comparison of viewing techniques. J Endod 1983; 9: 502-505.

11 Arnold L V. The radiographic detection of initial carious lesions on the proximal surfaces of teeth. Oral Surg Oral Med Oral Pathol 1987; 64: 232-240.

12 Mattesson O. Aspects of interpretation of contrast and detail in radiographs. Acta Radiol 1952; 38: 477-88.

13 Robinson P J A. Radiology's Achilles' heel: error and variation in the interpretation of the Rontgen image. Br J Radiol 1997; 70: 1085-1098.

14 Mileman P A, van der Weele L T. Accuracy in radiographic diagnosis: Dutch practitioners and dental caries. J Dent 1990; 18: 130-136.

15 Kaffe I, Gratt B M. Variations in the radiographic interpretation of the periapical dental region. J Endod 1988; 14: 330-335.

16 Hartmann E, Stieve F-E. Quality control of radiographic illuminators and associated viewing equipment. In: Technical and Physical Parameters for Quality Assurance in Medical Diagnostic Radiology. BIR Report 18, pp135137. London: British Institute of Radiology, 1989.

17 British Institute of Radiology. Part M1.3(m): measurement of the variation in intensity in one illuminator or a bank of illuminators. In: Assurance of Quality in the Diagnostic X-ray Department. p57. London: British Institute of Radiology, 1988.

18 SPSS for Windows. Release 9. 1998 SPSS Inc.

19 StataCorp. 1995. Stata Statistical Software: Release 4.0. College Station, TX; Stata Corporation.

20 Siegel S, Castellan N. Nonparametric statistics for the behavioural sciences. London: McGraw-Hill Book Company, 1988.

21 Wenzel A, Verdonschot E H. Some considerations in the evaluation of diagnostic tests in dentistry. Dentomaxillofac Radiol 1994; 23: 179-182.

22 Wenzel A, Hintze $\mathrm{H}$. The choice of gold standard for evaluating tests for caries diagnosis. Dentomaxillofac Radiol 1999; 28: 132-136.

23 Jensen T W. Image perception in dental radiography. Dentomaxillofac Radiol 1980; 9: 37-40.

24 Jenkins R. Radiographic photography and imaging processing. In Jenkins $\mathrm{R}$ (ed) Identification, Presentation and Viewing of Radiographs. Chapter 11. London: Wiley, 1983.

25 Lazarchik D A, Firestone, Heaven T J et al. Radiographic evaluation of occlusal caries: effect of training and experience. Caries Res 1995; 29: 355-358.

26 Kay E J, Watts A, Paterson R C et al. Preliminary investigation into the validity of dentists' decisions to restore occlusal surfaces of permanent teeth. Community Dent Oral Epidemiol 1988; 16: 91-94.

27 Kay E J, Knill-Jones R. Variation in restorative treatment decisions: application of receiver operating characteristic curve (ROC) analysis. Community Dent Oral Epidemiol 1992; 20: 113-117.

28 Goldman M, Pearson A H, Darzenta N. Endodontic success — who's reading the radiograph? Oral Surg Oral Med Oral Pathol 1972; 33: 432-437.

29 Goldman M, Pearson A H and Darzenta N. Reliability of radiographic interpretation. Oral Surg Oral Med Oral Pathol 1974; 38: 287-293.

30 Reit C, Hollender L. Radiographic evaluation of endodontic therapy and the influence of observer variation. Scand J Dent Res 1983; 91: 205-212.

31 Reit C, Grondhahl H-G. Endodontic retreatment decision making among a group of general practitioners. Scand J Dent Res 1988; 96: 112-117. 